\title{
The treatment of refractory angle-closure glaucoma in a patient with $X$-linked juvenile retinoschisis
}

Sancy Low, Ryian Mohamed, Michelle Ting, Andrew Webster, David Garway-Heath

X-Linked Retinoschisis (XLRS) is a common genetically determined form of macular degeneration affecting young males. Worldwide estimates of XLRS prevalence range from $1: 5000$ to $1: 20000 .{ }^{1}$ We report a thirty-nine-year-old man with refractory angleclosure glaucoma and genetically confirmed XLRS. The patient's retinal phenotype was atypical due to extensive exudative retinal detachments suggesting multiple mechanisms contributing to his glaucoma.

The patient presented with a two-day history of left eye pain and acutely reduced vision, on a background of a 9-month history of left sided hemicranial pain. His baseline visual acuity was counting fingers OD and 20/120 OS. On oresentations, visual acuity was hand movements OD and 20/300 OS. Prior IOP measuremets were $19 \mathrm{mmHg}$ OU, however, during this acute presentation IOP OS was $52 \mathrm{mmHg}$ and there was conjunctival injection, diffuse corneal oedema and a very shallow central and peripheral anterior chamber. Gonioscopy confirmed a completely closed angle. There was no iris or angle neovascularization, no posterior synechiae and no iris bombé. The crystalline lens was bulging anteriorly with visible glaucomflecken, but no frank phacodonesis. He patient was hypermetropic (OD $+3.00 /-1.00 \times 120$, OS $+5.00 /-$ $1.25 \times 25)$. The right eye was pseudophakic with inferior intraocular lens subluxation and he was treated for ocular hypertension. There was previously a dense white 
cataract in the right eye before cataract surgery was performed, and no history of ocular trauma. The left eye was phakic and was his better-seeing eye. Ophthalmoscopy showed a 360 degree retinal fold OD with a nasal tractional retinal detachment and a cup-to-disc ratio of 0.3 . There was no view of the left fundus due to the corneal opacification. Clinical and genetic analyses related to his XLRS disease had previously been undertaken by an Institutional Review Board approved study. He carried the NM_000330.3(RS1): c.574C>T (p.Pro192Ser) mutation.

The patient was managed medically with $1 \mathrm{gm}$ of intravenous acetazolamide, and topical timolol/ dorzolamide, latanoprost, iopidine and dexamethasone drops in his left eye. IOP OS decreased to $46 \mathrm{mmHg}$, with the corneal oedema clearing sufficiently to permit a YAG laser peripheral iridotomy. Following iridotomy, his IOP was $45 \mathrm{mmHg}$ and the iridocorneal angles remained closed. Anterior segment imaging and B-scan ultrasound excluded choroidal effusions. His chronic exudative retinal detachment, which had previously been noted in both eyes, remained unchanged. The axial lengths were $20.8 \mathrm{~mm}(\mathrm{OS})$ with an anterior chamber depth (ACD) of 1.6mm (OS), compared to $20.9 \mathrm{~mm}(\mathrm{OD})$ and $A C D$ of $4 \mathrm{~mm}$ with the subluxed lens (OD) suggesting possible zonular laxity in the right eye. Anterior segment imaging showed an anteriorly vaulted lens, hence the patient was instructed to lie supine and was given topical cyclopentolate $1 \%$. Six hours following this, the IOP decreased to $36 \mathrm{mmHg}$ but the iridocorneal angle remained closed. Urgent cyclodiode ablation of the ciliary body was performed the same day and the IOP decreased to $24 \mathrm{mmHg}$ two hours post-laser.

A week later, the pressure in the left eye was $7 \mathrm{mmHg}$ with ongoing treatment with topical cosopt bid, latanoprost qhs, iopidine tid and dexamethasone qid. Two weeks 
later, the IOP rose again to $43 \mathrm{mmHg}$ and he underwent a left lens extraction and IOL implantation without complications, and the patient was given a stat dose of atropine $1 \%$ post-operatively to reduce the risk of aqueous misdirection. Following surgery, his IOP decreased ed to $10 \mathrm{mmHg}$ to $14 \mathrm{mmHg}$ on without the need for glaucoma drops. His iridocorneal angle remained open. His vision improved to 20/100.

This case posed a difficult challenge given that this was a young patient with an 'only eye', whose acute rise in IOP was uncontrolled with medical treatment. Furthermore, he had a complex genetic retinopathy including XLRS but perhaps also another undiagnosed hereditary ocular condition that was responsible for his extensive bilateral exudative retinal detachments.

According to Ritch's anatomical classification of angle-closure (pupil block, plateau iris/ non-pupil block, lens-induced angle-closure and retro-lenticular causes $)^{2}$, this patient is unusual as his mixed mechanism angle-closure glaucoma included all four levels of block. Our patient underwent systematic investigation and treatment to exclude each of these levels of angle-closure. After failure of initial medical treatment, he underwent a laser peripheral iridotomy to relieve any pupil block component. An anterior segment optical coherence tomography scan was able to rule out plateau iris configuration and confirmed the clinical finding that the crystalline lens was bulging anteriorly. The B-scan ultrasound showed heavy calcification in the area of chronic exudative retinal detachment. 
At the molecular level, XLRS is due to mutations in the RS1 gene located on chromosome $\mathrm{Xp} 22^{3}$. RS1 codes for retinoschisin, a protein known to play an important role in cellular fluid balance, cellular adhesion and cell-cell interactions across bipolarphotoreceptor synapses. Absence of retinoshisin in XLRS patients subsequently leads to pathological extracellular fluid accumulation and reduced bipolar-photoreceptor integrity $^{3}$. XLRS patients are also at increased risk of developing ocular complications such as vitreous haemorrhage and retinal detachment ${ }^{4}$ but angle-closure glaucoma has not been reported previously.

Our patient had the NM_000330.3(RS1): c.574C>T (p.Pro192Ser) mutation. This RS1 mutation has been reported in other families ${ }^{4,5,6}$ but the specific phenotypic details relating to glaucoma such as axial length, refraction, and IOP were not available in the published reports. Interestingly, XLRS, is just one cause of macular retinoschisis, of which there are several known causes, such as retinitis pigmentosa, myopic degeneration and vitreo-macular traction. Of note, there are two case reports of angleclosure glaucoma in peripapillary schisis ${ }^{7}$ and macular schisis ${ }^{8}$. In both these cases, and our own, the mechanism of interaction between the retinal disorder and angleclosure glaucoma is unknown.

Lens surgery in the treatment of primary angle-closure glaucoma ${ }^{9}$ is gaining in popularity. There is an additional role for lens surgery in the treatment of secondary angle-closure glaucoma in the presence of an inherited retinal dystrophy. The present case demonstrates a systematic clinical and therapeutic approach to refractory angleclosure glaucoma, with good outcome following lens surgery. We suggest that patients with inherited retinal dystrophies presenting with headache or eye pain should 
undergo gonioscopic examination, so as to prevent the devastating visual loss which can occur in undiagnosed angle-closure glaucoma. ${ }^{10}$ 


\section{References}

1. George ND, Yates JR, Moore AT. X linked retinoschisis. Br J Ophthalmol 1995;79:697-702.

2. Ritch R, Liebmann JM. Role of ultrasound biomicroscopy in the differentiation of block glaucomas. Curr Opin Ophthalmol 1998;9:39-45.

3. Molday RS, Kellner U, Weber BHF. X-linked juvenile retinoschisis: clinical diagnosis, genetic analysis, and molecular mechanisms. Prog Retin Eye Res 2012;31:195-212.

4. Hiriyanna KT, Bingham EL, Yashar BM, et al. Novel mutations in XLRS1 causing retinoschisis, including first evidence of putative leader sequence change. Hum Mutat 1999;14:423-427.

5. Eksandh LC, Ponjavic V, Ayyagari R, et al. Phenotypic expression of juvenile Xlinked retinoschisis in Swedish families with different mutations in the XLRS1 gene. Arch Ophthalmol 2000;118:1098-1104.

6. Lesch B, Szabó V, Kánya M, et al. Clinical and genetic findings in Hungarian patients with X-linked juvenile retinoschisis. Mol Vis 2008;14:2321-2332.

7. Kahook MY, Noecker RJ, Ishikawa $\mathrm{H}$, et al. Peripapillary schisis in glaucoma patients with narrow angles and increased intraocular pressure. Am J Ophthalmol 2007;143:697-699.

8. Hollander DA, Barricks ME, Duncan JL, Irvine AR. Macular schisis detachment associated with angle-closure glaucoma. Arch Ophthalmol 2005;123:270-272.

9. Azuara-Blanco A, Burr J, Ramsay C, et al. Effectiveness of early lens extraction for the treatment of primary angle-closure glaucoma (EAGLE): a randomised controlled trial. Lancet 2016;388:1389-1397.

10. Low S, Davidson AE, Holder GE, et al. Autosomal dominant Best disease with an unusual electrooculographic light rise and risk of angle-closure glaucoma: a clinical and molecular genetic study. Mol Vis 2011;17:2272-2282. 